\title{
CrimRxiv
}

\section{Les sites de réseautage social et la présence des groupes criminels}

\section{Carlo Morselli ${ }^{1}$, David Décary-Hétu}

${ }^{1}$ Université de Montréal

Published on: Jul 08, 2020

DOI: 10.21428/cb6ab371.0bcce0c6

License: Creative Commons Attribution-NonCommercial-NoDerivatives 4.0 International License (CC-BY-NC-ND 4.0). 


\section{Résumé}

Ces dernières années, les médias ont véhiculé de plus en plus l’idée que des gangs de rue et des organisations criminelles se servent du Web pour diffuser leur image et pour faciliter leurs activités illicites. Si certains cas rapportés laissent présager qu'Internet et les sites de réseautage social, en particulier, sont utilisés par ces groupes pour recruter des nouveaux membres, très peu de recherches se sont attardées à évaluer et à comprendre le phénomène. La présente étude se propose d'examiner si une telle tendance est effectivement en place. Basée sur une recherche par mots- clés de plus de cinquante noms de gangs de rue, les trois principaux sites de réseaux sociaux (Twitter, Facebook et MySpace) ont été scrutés. Les résultats montrent que la présence de gangs sur les sites de réseautage social est liée principalement à la promotion d'un gang de rue ou de sa culture par ses membres mais sur une base individuelle. Rien n'indique que les visiteurs de ces sites sont soit trompés ou manipulés de quelque façon. Cependant, leur curiosité à l'égard de ces groupes et pour ceux qui partagent leurs observations et opinions, des signes de soutien sont évidents. Si les groupes criminels ne sont pas proactifs pour recruter des membres, les sites de réseaux sociaux leur servent à exposer leur mode de vie et leurs exploits à des personnes qui, dans le monde réel n'auraient probablement pu être rejointes.

\section{Introduction}

Plusieurs soutiennent que les organisations criminelles se sont tournées vers le Web et ses différentes ressources pour faciliter leurs activités illicites, pour se mettre en valeur et, éventuellement, pour recruter des membres. Le présent article vise à améliorer nos connaissances et notre compréhension sur la présence des groupes criminels dans les réseaux sociaux. Plus précisément, il s'intéresse à la gravité des menaces actuelles et futures que peut représenter l'usage de ces récentes technologies de communication par ces groupes. Le principal objectif de l'étude est de déterminer s'il existe bel et bien une problématique dans ce domaine.

La première section de l'article expose différents cas rapportés par les médias et les organismes d'application de la loi de la présence de gangs dans les sites de réseautage social. Lémergence des activités des gangs sur Internet est connue sous le nom de cyberbanging. La deuxième section traite des études qui se sont intéressées à ce phénomène et permet de le remettre en perspective et de voir au-delà des affirmations typiques relatives au recrutement en s'intéressant plutôt au sentiment d'appartenance et au mimétisme. La troisième section retrace l'historique des principaux sites de réseautage social actuels soit Twitter, Facebook et MySpace. Cette section est suivie d'une description de la méthodologie utilisée pour surveiller ces trois sites à l'aide d'une recherche par mots-clés. La cinquième section présente les résultats de cette analyse. Enfin, la dernière section formule les principales conclusions découlant de cette étude. 


\section{Cyberbanging?}

L'idée que l'apparition et l'évolution des sites de réseaux sociaux influencent le mode de fonctionnement des groupes criminels correspond avec la facilitation, un concept clé qui ressort des travaux sur le crime organisé. La facilitation renvoie aux différentes façons dont les intervenants, les situations et les contextes non criminels sont susceptibles de modifier les activités criminelles et les résultats du crime.

Dans ce domaine, les recherches se sont intéressées à de nombreux facteurs susceptibles d'attirer, de déclencher ou encore, d'influencer la formation de groupes criminels dans un territoire donné (Morselli, Turcotte, et Tenti 2010; Van Dijk 2007; Van de Bunt et Van de Schoot 2003; Jacobs 1999). Mais un contexte invariablement négligé dans ces études est celui d'Internet. Or, il semblerait que le crime organisé ait déjà fait son entrée dans le cyberespace.

En 2001, Williams notait une «tendance visible » dans des domaines aussi diversifiés que la fraude en valeurs mobilières, la cyberextorsion, le blanchiment d'argent, la propagation de virus informatiques et le réseautage des pirates informatiques. À l'heure actuelle, des tendances similaires sont également observées. Dans son plus récent rapport (2010), le Service canadien de renseignements criminels (SCRC) sonne l'alarme face à une augmentation de cybercriminels sophistiqués et de l'utilisation de logiciels malveillants ou de réseaux d'ordinateurs zombies, d'achats de produits sur Internet issus du marché noir (par exemple, les produits pharmaceutiques et les armes à feu), de vols d'identité et de fraudes informatiques (SCRC, 2010). Le rapport du SCRC fait également état de fraudeurs qui, par le biais de site de réseaux sociaux, recruteraient de nouveaux complices.

Certains pourraient prétendre que les technocrimes décrits par Williams et le SCRC sortent des paramètres traditionnels du crime organisé. Néanmoins, ces crimes tendent à refléter comment ces groupes criminels et leurs réseaux élargis optimisent l'utilisation du cyberespace à leur avantage.

Au cours des dernières années, les médias et les organismes d'application de la loi font état de cas où des gangs de rue et des organisations criminelles se sont servis des sites de réseautage social à différentes fins, que ce soit simplement pour la diffusion de leurs valeurs, de leurs exploits ou encore, pour recruter d'éventuels membres. Dans un article du Seattle PI portant sur les gangs de rue de Seattle et de Tacoma, Gutierrez (2006) décrit ce que les responsables des services de police entendent par cyberbanging ou de netbanging. Pour eux, il s'agit de l'utilisation des sites de réseautage social par les membres d'un gang pour « faire étalage de leurs exploits illicites, proférer des menaces ou rendre hommage à des membres tués ou incarcérés ». Alors que la plupart des spécialistes reconnaissent qu'il s'agit d'une forme de publicité, ils ne la conçoivent pas comme étant un processus de recrutement. Toutefois, l'un des spécialistes interrogés dans le cas de Seattle craint que ces sites servent éventuellement à des fins de recrutement. D’ailleurs, comme dans d'autres cas, on a souvent pensé que 
les interactions virtuelles sont à l'origine de rencontres dans la vie réelle susceptibles éventuellement de mener à une cooptation dans un gang. Dans son article, Vasquez (2008) est, quant à lui, beaucoup plus explicite sur les allégations de recrutement. Toutefois, il mentionne aussi clairement qu'elles émanent de sources policières qui soutiennent que les jeunes visitant les sites de réseautage social s'exposent aux expériences de membres de gangs. Ainsi, ils risquent à leur insu de devenir d'éventuelles recrues. Le Daily Star de Londres rapporte un cas éloquent de recrutement de la part d'un groupe criminel par l'entremise de réseaux sociaux. Un visiteur d'un site web a expressément été invité à se joindre à la Mafia sicilienne. Il semblerait que le jeune homme âgé de 18 ans se soit inscrit à un groupe Facebook dédié au réputé chef de la mafia Bernardo Provenzano. Il aurait ensuite été approché par un homme mentionnant être à la recherche de Siciliens pour faire la collecte de paiements de protection. Le jeune homme a refusé poliment et effacé son profil, mais il semble que certains de ses amis aient accepté l'offre et soient devenus impliqués dans ce commerce illicite.

\section{Les gangs, les réseaux et le monde virtuel}

Si certaines données non scientifiques semblent indiquer que les gangs se tournent vers les sites de réseautage social pour recruter de nouveaux membres, peu de recherches ont porté spécifiquement sur l'utilisation exacte d'Internet par les gangs de rue et les groupes criminels affiliés au crime organisé. Decker, Van Gernert et Pyrooz (2009) ont remarqué que l'expansion de ces groupes en Europe a été en partie attribuable à la diffusion de la culture des gangs sur Internet. Ils ont cependant bien mis en garde de ne pas restreindre l'explication de ce phénomène à cette seule cause. En effet, Internet n'est pas le seul moyen de communication véhiculant ce genre d'images; la télévision, les films, la musique et les vidéos servent abondamment à diffuser la vague populaire du hip-hop et le mode de vie des gangs de rue. Womer et Bunker (2010) ont étudié dans quelle mesure Internet s'est imposé comme plate-forme de diffusion des gangs de rue. En effectuant des recherches par mots-clés, ils ont évalué l'utilisation des sites de réseautage social par les narcogangs mexicains. Ainsi, ils ont constaté que les gangs affiliés aux Surenos se servaient des sites de réseautage social pour vanter leurs exploits et diffuser leur image. Ils ont aussi observé la présence d'autres gangs, notamment le MS-13 et le 18th Street. Les sites Web dédiés à ces gangs renfermaient des photos des membres portant des armes à feu, faisant des signes de main et exhibant leurs tatouages. À l'instar de la présente étude, Womer et Bunker (2010) ont également tenté de vérifier si les gangs recrutaient de nouveaux membres par le biais de ces sites. Ils ont relevé que certains organismes, tels que le FBI, considéraient cette glorification exacerbée des gangs dans les pages des membres comme étant une méthode de recrutement (p. 85). Toutefois, leur recherche par mots-clés n'a généré aucune donnée probante étayant quelque forme que ce soit de stratégie de recrutement proactif.

D'innombrables travaux ont documenté l'importance de reconnaître la formation et l'activité des gangs comme un phénomène de réseau. De tels travaux constituent une solide pierre d'assise pour la 
présente étude. Les résultats des recherches antérieures nous ont incités à remettre en cause l'existence d'une sous-culture de gang organisé. Les travaux de Klein et Crawford (1967) ont pavé la voie aux études en réseau de l'organisation des gangs de rue. Ils ont étudié les interactions entre les membres du noyau d'un gang de Los Angeles et les membres en périphérie qui participaient à un programme d'intervention. Les chercheurs ont constaté une absence de cohésion, un leadership versatile et une hiérarchie des membres. Même après plus de trois décennies de recherche sur les gangs, ces constats demeurent toujours aussi pertinents (voir, par exemple, Weisel, 2002; Fleisher, 1995; Skolnick, Bluthenthal et Correll, 1993) et devraient alimenter tout exercice de réflexion par rapport à la présence des gangs à l'ère d'Internet. À bien des égards, la structure du gang est grandement cloisonnée et les approches axées sur les réseaux l'ont, dans une grande mesure, confirmée. L'un des modèles de recherche le plus important issu de cette approche est celui utilisé dans le cadre du projet contre les armes à feu de Boston. Le Boston Gun Project est une initiative en résolution de problème élaborée pour contrer la propension à la violence croissante chez les jeunes de la ville. Kennedy, Braga et Piehl (1997) ont mené des entrevues et effectué des exercices de représentation cartographique auprès de spécialistes des gangs de rue du service de police de Boston, des agents de probation et des travailleurs sur le terrain. Ce travail visait à représenter graphiquement les réseaux d'alliances et de conflits entre les gangs de rue de Boston. Les auteurs ont constaté que l'organisation des gangs n'était pas fortement centralisée mais les gangs qui se sont révélés au centre des réseaux étaient aussi ceux considérés comme les plus criminalisés.

D'autres recherches ont repris le flambeau là où le Boston Gun Project l'a laissé. Tita, Riley, Ridgeway, Grammich, Abrahamse et Greenwood (2003) ont procédé à une analyse de réseau pour évaluer l'efficacité de diverses stratégies visant la réduction de la violence liée aux gangs à Los Angeles. En consultant des responsables des forces policières et des membres de groupes communautaires, les auteurs sont parvenus à dresser le portrait du réseau des rivalités et de violence parmi 29 gangs de rue. Les résultats ont montré comment les agressions individuelles et collectives étaient dispersées et contenues dans une aire géographique divisée par l'autoroute San Bernardino. Les gangs rivaux ne traversaient l'autoroute que par un seul pont. Encore là, la structure de gang semblait manquer de cohésion.

En s'inspirant du Boston GunProject, McGloin (2005) a mené, à Newark, une recherche en collaboration avec l'escouade antigang du New Jersey. Elle a amorcé ses travaux en tentant de repérer les points névralgiques de l'activité et des interactions des gangs à Newark et ses environs. Les résultats de 32 groupes de discussion composés de policiers spécialistes ayant identifié les membres des gangs et leurs collaborateurs respectifs ont révélé que quatre grands gangs (les Bloods, les Crips, les Latin Kings, et les Netas) dominaient Newark. Cependant, au lieu d'être concentrés dans les secteurs névralgiques, ils étaient disséminés sur tout le territoire. De façon générale, aucun réseau dominant n'a été observé. 
Plus récemment, Morselli (2009) a analysé les interactions entre les membres d'un gang et des non membres dans le cadre d'une opération policière à Montréal visant un réseau de distribution de drogue. Il a constaté que lorsque les membres d'un gang étaient présents dans un réseau, ils agissaient surtout de façon autonome et n'étaient pas les principaux membres du réseau. Le fait que les membres d'un gang ne soient pas directement au centre de l'organisation du gang n'est pas contradictoire en soi. Par exemple, Katz, Webb et Schaefer (2000) ont constaté que les personnes ne faisant pas partie d'un gang sont souvent des acteurs influents et importants dans des organisations qui, au départ, étaient considérées comme affiliées à des gangs. À bien des égards, ces recherches confirment l'expérience vécue par l'un des sujets interrogés précédemment par Klein (1971) : “We got no leaders, man. Everybody's a leader, and nobody can talk for nobody else" (p. 96). La nature individualiste des gangs de rue est susceptible de se révéler encore davantage dans le contexte d'Internet. Tout en reconnaissant le caractère communautaire des interactions sur Internet, Wellman (2004; 2001) mentionne que cette nouvelle frontière commune évolue en grande partie sous l'égide d'un individualisme réseauté (networked individualism). En ce sens, Wellman sous-entend que même si les personnes se réunissent de plus en plus dans des communautés du Web, la vitesse, la facilité et le caractère éphémère des relations qui les rendent possibles en font un phénomène individualisé où l'individu (et non le groupe ou le réseau) se trouve au centre de son propre univers. Même si l'individu est à l'avant-scène, Burt (2010) ajoute que, tout comme dans le monde réel, le monde virtuel est sujet aux mêmes processus d'homophilie sociale qui réunissent les individus. Cependant, bien que les personnes partageant des goûts, des intérêts et des réalisations similaires puissent converger grâce à des réseaux de communication virtuels, Burt insiste aussi sur le fait que la confiance et la réputation sont nettement distinctes des expériences vécues dans le monde réel. Il prétend que plusieurs causes pourraient aussi entraîner la disparition de ces dispositifs usuels. Dans le cas de la présence des gangs de rue dans Internet, en particulier sur les sites de réseautage social, ces constats suggèrent: 1) que les interactions sont plus susceptibles de se produire autour des membres et non autour du gang luimême; 2) qu'en raison du faible niveau de confiance et de réputation entre internautes, l'idée que les membres d'un gang soient réellement en train de recruter de nouveaux membres ou des complices est peu probable, les membres se méfiant d'un tel risque.

Il semble donc plus probable que nous assistions à l'émergence d'un processus d'homophilie sociale dans le cadre duquel des personnes partageant des goûts et des modes de vie similaires convergent vers un même site. Les membres d'un gang qui utilisent ces sites peuvent signaler leur appartenance et inciter les visiteurs à joindre le gang. Gambetta (2009) définit ces signaux comme "the stuff of purposive communication" et "any observable features of an agent that are intentionally displayed for the purpose of altering the probability the receiver assigns to a certain state of affairs or event" (p. xv). Toutefois, il reste à établir si les membres du gang attirent intentionnellement les aspirants ou les recrues potentielles ou s'ils font simplement exprimer leur liberté, de manière aussi provocante soit- 
elle. Pour l'instant, ces expressions prêtent le flanc à une grande marge d'interprétation. L'image et l'interprétation relatives à ce phénomène sont aussi abordées par Felson (2006) dont les travaux sur les stratégies de signalement doivent être également considérés. Felson (2006) a constaté que bon nombre des groupes criminels ou gangs de rue moins réputés adoptent les signes de la main, les couleurs, le jargon, les vêtements et même le nom de gangs réputés afin de rehausser leur image. Ce mimétisme est au cœur de bon nombre de mythes et de stéréotypes entourant les gangs de rue véhiculés dans les médias et le milieu de l'application de la loi. En effet, même dans les cas où la stratégie de signalement est intentionnelle, il se peut que le gang ou le groupe derrière le message ne soit pas établi de façon sérieuse.

Étant donné la grande part d'ambiguïté entourant les motifs précis expliquant pourquoi les gangs de rue et d'autres organisations criminelles ont recours aux médias sociaux, la présente étude se propose d'étudier le phénomène en mesurant directement le trafic d'accès à ces sites. Avant de décrire la méthodologie de recherche, un bref aperçu des trois sites de réseautage est présenté pour permettre de comprendre comment et pourquoi ces canaux de communication sont devenus, au-delà de la problématique des groupes criminels, une source immensément populaire.

\section{L'émergence des médias de réseautage social}

Les sites Web de réseautage social ont beaucoup évolué depuis leur apparition dans le milieu des années 1990. Le premier service du genre portait le nom de Bulletin Board System (BBS) (Zhongbao et Changshui , 2003). Ces systèmes étaient de simples ordinateurs auxquels un nombre limité de personnes pouvaient se connecter afin d'échanger des messages ainsi que des fichiers. Leur interface, très simpliste (contenant exclusivement $d u$ texte), était populaire auprès d'amateurs $d$ 'informatique clandestins (Craig, 2005). Dans le milieu des années 1990, l'Internet et de nouvelles communautés ont graduellement remplacé les BBS. Geocities et Tripod ont été parmi les premiers à transposer l'idée de collectivités dans le monde virtuel en offrant à leurs utilisateurs une façon de trouver des ressources sur tous les sujets. Lancé en 1997, le premier véritable site de réseautage social portait le nom de SixDegrees (Boyd et Ellison 2007). Ce service était intuitif en plus d'être gratuit. Cinq ans plus tard, en 2002, c'était au tour de Friendster de faire son apparition. Ces sites ont été les premiers à offrir à leurs utilisateurs la possibilité de créer des profils en ligne grâce auxquels ils pouvaient communiquer avec des amis et les amis de leurs amis. En quelques mois, des sites Web comme Friendster comptaient déjà plus de trois millions d'utilisateurs (Rivlin, 2006).

La réussite de ces premiers sites n'est pas passée inaperçue. De jeunes entrepreneurs ont reconnu le potentiel que représentaient les sites de réseautage social et, en quelques jours, ont créé MySpace. Semblable à Friendster, les utilisateurs affichent leur profil et ils peuvent modifier la présentation de leur page afin de refléter leur personnalité. Il offre également aux musiciens la possibilité de faire connaître leur musique à des millions de personnes. Le lecteur de musique de MySpace est 
maintenant connu des amateurs de musique de partout dans le monde. Ce site de réseautage social possède même sa propre étiquette maison. Le succès de MySpace a été instantané (Boyd et Ellison, 2007). En 2006, il s'agissait du site de réseautage social le plus populaire sur Internet. Cette réussite était en grande partie attribuable au fait que tous les profils étaient, par défaut, accessibles à tous. Cette caractéristique a permis de s'assurer que les internautes pouvaient aisément se retrouver les uns les autres. Cependant, une telle ouverture n'a pas été sans problème. MySpace a été critiqué pour la facilité avec laquelle les harceleurs étaient en mesure de retracer leurs victimes (ex.cyberintimidation). Même si MySpace se concentre désormais sur la promotion de la musique, il regroupe encore un nombre important d'utilisateurs qui échangent leurs opinions personnelles ou des images et d'autres qui communiquent avec des amis et des membres de leur famille.

Les problèmes qu'a connus MySpace ont incité bon nombre d'utilisateurs à migrer vers un site de réseautage social plus en vogue : Facebook. Ce site offrait au départ des profils privés par défaut en plus d'un riche environnement pour demeurer en contact avec son réseau de connaissances par le truchement de messages, de photographies, de vidéos et de jeux. Même si ce site de réseautage social était limité au départ aux étudiants des grandes universités américaines, il a progressivement ouvert ses portes à tous les individus âgés de plus de 13 ans. À l'instar de MySpace, les forces de Facebook sont rapidement devenues ses faiblesses. Le site web est devenu de plus en plus achalandé grâce à de nouvelles applications, son centre de communication et son contenu audio et vidéo. Avec le temps, l'emphase initiale sur le respect de la vie privée a été délaissée pour plaire aux annonceurs toujours plus nombreux et insistants. Facebook a donc modifié ses règlements internes sur la vie privée rendant ainsi publics plusieurs informations et médias personnels. Devant l'opposition de ses utilisateurs, le réseau social a dû faire partiellement marche arrière, mais c'était trop peu et trop tard. Ces difficultés ont incité un grand nombre d'utilisateurs de Facebook à migrer vers le dernier venu du domaine des médias sociaux, Twitter.

Twitter a été lancé en 2006. Contrairement à Facebook et MySpace, le site a intégré dès ses débuts la technologie des téléphones cellulaires, le rendant ainsi extrêmement accessible et facile d'accès. La caractéristique unique à Twitter est le gazouillis, un message de 140 caractères communiqué à des lecteurs partout dans le monde. Au départ, ces gazouillis étaient très simples, mais au fil du temps, une nouvelle forme d'art a émergé - celle de la concision. En effet, les utilisateurs de Twitter se livrent à une intense compétition afin de communiquer le message le plus percutant en utilisant le moins de caractères. Les adeptes de technologie ont été les premiers à adopter le service lorsqu'il a été mis en ligne et ils ont apprécié l'interaction directe entre les individus - aucune application, photographie, cadeau ou autre distraction ne vient polluer l'interface qui se concentre sur les messages échangés entre une personne et son réseau de contacts. Tous les gazouillis sont publics par défaut. Une telle ouverture crée un fort sentiment d'appartenance entre les membres de ce site de réseautage social. 


\section{Méthode}

À l'instar de Womer et Bunker (2010), une recherche par mots-clés a été effectuée pour déterminer si les gangs ou les membres individuels se servent des réseaux sociaux pour recruter de façon proactive de nouveaux membres, communiquer avec les membres et collaborateurs existants d'un gang commun, ou diffuser leur image en quête de soutien de personnes sensibles à ce type de signalement. La recherche a été limitée aux utilisateurs actifs dans les trois principaux sites soit Facebook, MySpace et Twitter. Comme le démontre une enquête menée récemment, ces trois sites sont les plus populaires à l'heure actuelle (Nielsen Company 2010).

Nous avons consulté des forums de discussion, des journaux et des sites web gouvernementaux pour trouver les noms de gangs de rue ainsi que d'organisations criminelles actifs au Canada et à l'étranger. Les noms les plus fréquemment cités ont été sélectionnés afin de créer la liste finale de mots-clés :

Bloods, Crips, Crack Down Posse, Kazee Brezze, MS-13, Mara Salvatrucha, Bo-Gars, Bad Boys, XVIII, 18th Street, Playboy Gangster Bloods, West Side Ballaz Gang, Dark Family, Blood For Life, Black Eyed Crips, Outlawz Nation Cripz, Baby Boy Crips, Blue 450 z Crips, Blue 450 z Crips, West Sidaz, Young Pimp Ganster Crips, Gun Clappin Crips, StLaurent Crips, Asian Young Bloods, Asian Blue Crips, Jamestown Crips, MNM (Mother Nature's Mistakes), Mount Olive Crips, Ghetto Boys, NBC (Net Born Crips), Junior Crips, 18 Buddhas, Ak Karman, Malvern Crew, Galloway Boys, Regent Park Crew, One Love Crew, Ruthless Russians,Ardwick Blood Crew, McCormack Boys, Hells Angels, Asian Boys, 14k Triad, Almighty Vice Lord Nation, Big Circle Gang, Mara Salvatrucha, Latin Kings, Luen Group, Mickey Cobras, Show er Posse, VVT, Wo Shing Wo, West End Gang, Wah Ching, Red Scorpions, Independent Soldiers.

Certains des noms de la liste contiennent des mots qui, dépendant du contexte, peuvent être associés ou non à des organisations criminelles (p. ex., Blood for Life, 18th Street). Afin d'éviter les associations erronées, nous avons ajouté des termes tels que gang, drogues, violence et crime à nos requêtes. Cette approche nous force à nous limiter aux groupes criminels qui utilisent un nom publiquement. Afin de réduire les chances d'identification, certains d'entre eux préfèrent ne pas adopter d'appellation. Ces groupes ne peuvent donc pas être détectés au moyen de cette méthode de recherche. Aussi, chacun des sites possèdent des particularités qui devaient être considérées pour mener à bien la recherche. Les sites ont des fonctions de recherche et une interface différente, n'offrent pas tous, par défaut, le même niveau de protection des renseignements personnels et leur quantité d'informations varient. Les stratégies de recherche ont donc été adaptées à chacun de ces réseaux.

Tel que mentionné précédemment, Facebook a grandement réduit le niveau de protection des renseignements personnels au cours des dernières années. Cela a eu pour effet de générer une mine d'or d'information pour les chercheurs qui peuvent maintenant puiser dans des groupes, des activités, des images et des profils qui, auparavant, se voulaient privés mais qui sont désormais accessibles à 
tous, à l'insu des utilisateurs ou du moins sans leur consentement explicite. La recherche par mots-clés sur Facebook a été réalisée à l'aide de son moteur interne de recherche. Étant donné que les profils de Facebook sont privés par défaut, il aurait été futile de tenter de les indexer. Nous avons plutôt opté pour les pages et les groupes de Facebook. Les pages sont habituellement des profils ouverts créés pour soutenir une idée ou une organisation. Les personnes peuvent ensuite dire qu'elles «aiment» cette page et créer un lien vers elle. Les groupes sont plus riches en contenu et les membres peuvent discuter de divers problèmes personnels et sociaux, créer des activités et échanger des médias. Les utilisateurs peuvent devenir des membres et le nom des créateurs de ces groupes sont affichés sur la page. Les pages et les groupes peuvent contenir des messages, des discussions, des déclarations, des images, des vidéos et des activités accessibles au grand public.

À l'opposé de Facebook, les profils de MySpace sont publics par défaut. Les utilisateurs peuvent, s'ils le désirent, empêcher des inconnus d'accéder à leur page, mais la grande majorité d'entre eux utilisent les réglages par défaut. Nous avons donc été en mesure d'effectuer une recherche parmi les millions de profils disponibles. Comme dans le cas de Facebook, nous avons tenté d'utiliser le moteur interne de recherche en nous concentrant sur les images et les vidéos associés aux mots-clés. Au moment de réaliser cette étude, le moteur interne de recherche nétait malheureusement pas des plus conviviales; les résultats ne sont pas clairement indexés et plusieurs profils ne sont pas détectés adéquatement. Nous nous sommes donc tournés vers la fonction de recherche «InSite » de Google. Cet outil permet de limiter des recherches à un site en particulier - dans ce cas: myspace.com. Nous avons donc utilisé cet outil afin d'identifier les profils reliés à nos mots-clés.

Twitter offre les données les plus accessibles de tous les sites de réseautage étudiés. Tous les messages affichés dans le site sont publics et peuvent faire l'objet de recherches approfondies. Le site offre une fonction de recherche intégrée claire et utile qui donne accès à tous les « gazouillis » communiqués au cours des dix derniers jours. La limite de Twitter provient essentiellement du petit nombre de caractères qui n'offre que des renseignements très limités à chaque fois.

\section{Résultats}

Il semble de notoriété publique que les membres de gangs de rue et de groupes criminels utilisent les nouvelles technologies pour commettre des crimes, communiquer et recruter de nouveaux membres. Les sites de réseautage social apparaissent alors être l'outil privilégié. La présente étude suggère que si le recrutement de nouveaux membres n'est pas si répandu, ces groupes ont une forte visibilité dans le cyberespace et ce, encore plus de ce qui est rapporté dans les journaux. Les trois sites étudiés ont généré trois ensembles de données très distinctes que nous analyserons séparément. 


\section{Twitter}

Twitter est le plus jeune des sites de réseautage social et apparait comme étant le moins utilisé par les groupes criminels. La recherche par mots-clés n'a généré que très peu de données probantes indiquant la présence des groupes criminels. Comme le montre le tableau 1, nous avons constaté la présence de huit groupes. Certains mots-clés (18th Street, Asian Boys, Bad Boys, Dark Family, VVT et XVIII) ont donné lieu à un nombre important de résultats mais qui ne se rapportent pas à des groupes criminels. Ainsi, les résultats obtenus à l'aide du mot-clé VVT étaient essentiellement liés à des discussions traitant de véhicules tout terrain. D’autres résultats étaient, pour leur part, sans intérêt. Un utilisateur a, par exemple, simplement publié un lien vers un article de journal traitant du gang des $14 k$ Triad. Aucune indication ne permettait de déterminer si l'auteur du gazouillis était membre ou non de ce gang. Des utilisateurs ont gazouillé leur soutien à l'égard de diverses organisations et des aspects les plus stéréotypés du mode de vie criminel en utilisant un langage blasphématoire, en dégradant les femmes et en mettant l'accent sur l'importance de l'argent.

\section{[INSÉRER LE TABLEAU 1 ICI]}

Bien que la plupart des groupes ne s'affichent pas publiquement sur Twitter, certaines organisations ont laissé une empreinte plus visible. Les Hells Angels comptent parmi les groupes les plus notoires sur Twitter. Ceci n'est pas surprenant en soit puisque les Hells Angels possèdent également une corporation légale et que bon nombre des chapitres de l'organisation ont des pages officielles où les membres discutent de leur mode de vie et d'événements reliés au groupe. En outre, cette organisation jouit d'un grand nombre d'abonnés et de partisans, en particulier chez les amateurs de moto. Bon nombre de gazouillis proviennent de supporteurs qui font l'éloge des Hells Angels. En ce qui a trait au recrutement de la part des Hells, aucun signe na été constaté. Une seule mention provient d'un utilisateur qui demandait à un de ses contacts s'il prévoyait joindre les rangs des Hells Angels. Dans ce cas-ci, il est difficile de déterminer le niveau d’engagement proposé: «Est-ce qu'on se joint aux Hells? Mieux vaut régler ça! »

Quelques gangs de rue sont actifs sur Twitter. Le gang jamaïcain Shower Posse semble attirer davantage l'attention. Il est fort probable que le documentaire télévisé de la chaîne A\&E «Gangland» ne soit pas étranger à cet intérêt. Un membre du gang met de l'avant son mode de vie débauché et louange le groupe tout en parlant d'alcool, de drogue et de femmes. Cette mise en valeur d'un mode de vie débridé est très présente chez les membres du MS-13 ou Mara Salvatrucha. La toile de fond de leur page présente souvent des images d'argent, d'armes à feu et les couleurs du gang.

Certains utilisent Twitter pour montrer leur fort sentiment d'appartenance à un gang. Des pages de ce type existent pour les Latin Kings, les Crips, et les Bloods qui font tous étalage de leur image et de leur mode de vie. Nous avons également répertorié un petit nombre de profils privés liés à ces gangs, mais 
pour lesquels les messages n'étaient malheureusement pas accessibles. Toutefois, les caractéristiques visuelles de ces profils semblaient similaires à ceux auxquels nous avons eu accès. Dans l'ensemble, le principal motif sous-jacent à l'utilisation de ces réseaux est la diffusion de la culture du gang.

Twitter demeure un service relativement nouveau qui a connu un élan de popularité au cours des derniers mois. Il n'y a pas si longtemps, les utilisateurs comptant des centaines de milliers d'admirateurs étaient peu nombreux. Les membres de Twitter semblent être habituellement plus âgés et plus sophistiqués, ce qui correspond probablement moins au profil typique des membres de gangs de rue ou des organisations criminelles. Il n'est donc pas si étonnant de constater l'absence des plus petits groupes et que seuls les principaux joueurs se démarquent dans une certaine mesure. Nous avons dénombré moins de dix profils pertinents pour chacune des organisations présentes, ce qui est très faible compte tenu du fait que des millions de personnes utilisent Twitter. Malheureusement, le moteur interne de recherche est relativement limité et certains mots-clés, comme par exemple 18th Street, génèrent un nombre élevé de résultats non pertinents. En outre, une multitude de gazouillis se perdent dans le nombre élevé de gazouillis liés à d'autres référents plus courants. Même avec un nombre aussi élevé de gazouillis, des liens vers les gangs n'ont pas pu être relevés. Se conformant à la limite « créatrice » de 140 caractères, bon nombre des messages rédigés par les membres de gangs ou leurs contacts sont bel et bien concis, mais essentiellement incompréhensibles pour tout néophyte qui n'est pas habitué à leur jargon.

\section{Facebook}

Il existe plusieurs similitudes entre les résultats de Twitter et de Facebook. Le Tableau 2 présente le nombre global d'admirateurs ou de membres de chaque gang tel que répertorié lors de nos recherches sur Facebook. Tout comme sur Twitter, une minorité de gangs est sur Facebook mais le nombre de groupes actifs repérés lors de nos analyses est beaucoup plus élevé que dans le premier cas (18 par rapport à 8).

Les pages de la quasi-totalité de ces groupes affichent des images de membres tenant des armes à feu, arborant leurs couleurs et faisant des signes de main. Fréquemment, leurs pages contiennent des images de scènes violentes. Il semble que l'objectif visé est de montrer la force, la taille et la détermination du gang. Le mur des pages Facebook sont, la plupart du temps, remplis de messages élogieux sur le gang. Sur certaines pages, plusieurs utilisateurs cherchent à mettre en valeur la puissance de tel ou tel gang (par exemple, les pages des Crips et des Bloods). Les discussions soutenues et suivies sont extrêmement rares. Seules les pages des Hells Angels renferment des échanges significatifs et pertinents portant principalement sur des motards arrêtés (à quel point il est injuste qu'ils soient en prison) et sur la manière d'interagir avec les forces de l'ordre (quoi faire lors d'une arrestation ou d'un interrogatoire). Dans un cas (une page des Bloods), les utilisateurs sont allés jusquà rendre public le prochain rassemblement de leur gang en incluant l'heure, le lieu et les personnes 
invitées. Cette pratique exceptionnelle est soit la preuve d'une bravade importante ou encore d'une totale négligence de la part de ce groupe criminel.

Certains recoupements entre les résultats de Twitter et de Facebook ont été constatés. À l'exception des Almighty Vice Lord Nation, tous les groupes présents sur Twitter le sont aussi sur Facebook. Le nombre d'admirateurs ou de membres varie considérablement allant de 297 dans le cas des Shower Posse à 14775 dans le cas des Hells Angels. De nouveau, il semble que les groupes bien établis à l'échelle internationale attirent le plus de visiteurs. Même si ces chiffres peuvent sembler impressionnants, il faut se garder de tenter d'évaluer le nombre de membres officiels en se basant sur le nombre d'admirateurs Facebook. Il n'existe en effet aucune façon de vérifier si ces admirateurs sont de véritables membres du groupe ou de simples sympathisants virtuels sans avoir accès à leur profil privé. Il est fort probable que ces personnes soient, pour la plupart, curieuses ou admiratives face à ces organisations criminelles. En outre, il ne serait pas surprenant de découvrir que, parmi ces personnes, se cachent des membres des forces policières qui surveillent les communications en ligne entre les membres de gangs de rue.

\section{[INSÉRER LE TABLEAU 2 ICI]}

Bon nombre de groupes avaient aussi des pages dont le contenu était lié à Wikipédia et d'autres services en ligne. Ces services présentaient habituellement l'historique du gang, les réalisations de ses membres les plus visibles et une estimation de leur taille et de l'étendue de leur pouvoir. Peu de contenu aurait pu être décrit comme étant un effort de propagande ou de recrutement.

Facebook est surtout utilisé pour diffuser de l'information sur les gangs et pour faire étalage de leur pouvoir par le truchement d'images, de leurs couleurs et d'armes à feu. Hormis un profil affiché par les Bad Boys, peu de messages pouvant être interprétés comme des tentatives de recrutement ont été relevés. Toutefois, les gangs insistent sur la transmission de leur message collectif et de leur raison d'être. Pour la plupart, la présence du gang sur un site de Facebook est un effort pour se confirmer comme faisant partie de 1' élite. Des messages dépassant les prétentions de célébrité d'un gang ont été répertoriés dans seulement un petit nombre de pages. Par exemple, la présence des Hells Angels sur Facebook visait plus particulièrement à prétendre qu'ils sont un club de motards légitime n'ayant aucun lien criminel. On y a aussi trouvé quelques pages consacrées à des membres arrêtés partout dans le monde et des messages servant à informer les admirateurs et les membres sur la façon d'aider à dénoncer les injustices perçues.

\section{MySpace}

Parmi les sites de réseautage social étudiés, MySpace est celui qui attire le public le plus jeune. Il s'agit, par ailleurs, du plus ancien d'entre eux. Ces deux facteurs nous amènent à penser que MySpace sera le plus approprié des sites de réseaux sociaux pour retracer la présence de membres 
d'organisations criminelles. Le Tableau 3 confirme cette intuition et présente le nombre total d'amis ainsi que le nombre de pages vues (lorsque disponible) pour chacune des organisations.

\section{[INSÉRER LE TABLEAU 3 ICI]}

Bien que le nombre de gangs répertoriés sur MySpace soit légèrement inférieur à celui de Facebook (16 contre 18), les profils de MySpace comptent beaucoup plus d'amis que d'admirateurs ou de membres sur Facebook. Certains gangs absents de Facebook et Twitter (West End Gang, Malvem Crew, West Sidaz) jouissent d'une présence importante sur MySpace. L'image projetée semble tout aussi importante sur MySpace que sur Facebook. Tous les gangs mettent en ligne une multitude de photos sur lesquelles les membres brandissent des armes à feu, leurs couleurs ou font des signes de main. Nous avons dénombré plus d'images sur MySpace que sur Facebook et bon nombre de celles-ci sont davantage incriminantes. À titre d'exemple, une page des Wah Ching présente une image d'arme automatique entre les mains d'une personne se cachant derrière un drapeau rouge. Bon nombre d'images similaires (membres de gangs avec de l'argent, des armes dangereuses et des drapeaux) ont aussi été répertoriées sur les profils des Bloods for Life, des Crips, et des Asian Boys. Tout comme sur Facebook, bon nombre de ces pages sont remplies de louanges de la part des admirateurs et des amis du gang. À la différence de sur Facebook, MySpace compte plusieurs agents provocateurs qui cherchent à créer des conflits entre les organisations en utilisant la section des commentaires pour lancer des insultes (par exemple, les Crips sur les sites des Bloods).

Contrairement à d'autres sites de réseautage social, il existe sur MySpace une légère volonté de recrutement. Bon nombre de gangs y présentent des vidéos promotionnels de leurs membres tirant avec des armes à feu, battant d'autres membres du gang, effectuant des signes de la main, vantant le nombre de membres, arborant leurs tatouages et exprimant leurs croyances. Dans certains cas, les profils comprennent aussi des messages à l'intention des nouveaux membres et des nouvelles recrues. C'est par exemple le cas des Asian Boys, des Bad Boys, des Wah Ching, des MS-13, et des Crips. L'un des profils présente d'ailleurs une image de l'Oncle Sam cherchant à recruter de nouveaux membres pour le MS-13 (http://www.myspace.com/elmara8). En fait, les profils de MySpace semblent accorder plus d'importance au fait de projeter une image de force et de pouvoir de l'organisation

Peu de profils affichent le nombre de visiteurs mais parmi ceux qui le font, ce sont les groupes internationaux comme les Hells Angels, 18th Street et Wah Ching qui sont le plus suivis. Sur MySpace, les Hells Angels sont dans une classe à part tout comme sur Facebook. Le club de motards compte vingt fois plus d'amis que tout autre gang ou organisation. Encore une fois, les membres réclament une plus grande justice à l'égard de leurs confrères.

De toute évidence, MySpace est le site de réseautage social où les membres de groupes criminels sont les plus actifs. Les membres semblent aussi plus enclins à discuter de leur mode de vie et de leur lien 
au gang. C'est aussi le site dans lequel les conversations et les commentaires sont les plus nombreux et actifs. Enfin, il s'agit du seul site dans lequel des vidéos dont le contenu est très dur sont diffusés.

\section{Conclusion}

Pour plusieurs, il peut sembler naturel que les membres de gangs utilisent des sites comme Twitter, Facebook et MySpace comme tout autre citoyen. Pour d'autres, il est difficile de croire que les gangs sollicitent activement des personnes par des moyens aussi ouverts et pouvant être facilement surveillés. Mais il est possible que les membres de gangs utilisent de tels sites à des fins personnelles et non pas nécessairement pour recruter directement de nouveaux membres.

La recherche par mots-clés effectuée dans le cadre de la présente étude montre clairement que les gangs se servent des réseaux sociaux essentiellement pour promouvoir la culture générale du gang, ou de la rue, au moyen de communications individuelles. Dans la plupart des cas, les sites sont conçus et gérés par les membres et les collaborateurs qui affichent leur allégeance envers des groupes réputés comme les MS-13, les Crips, les Bloods, ou les Latin Kings. Ces quatre gangs sont les plus visibles dans les sites de réseautage social. Tout aussi visibles, les Hells Angels se démarquent toutefois des autres groupes par une présence en ligne en tant que chapitres ou de groupes plutôt que sur une base individuelle. Contrairement à la majorité des gangs de rue, les Hells Angels ne font pas étalage de leurs exploits criminels ou violents. De fait, ils sont plus susceptibles de diffuser des aspects non criminels du groupe et des problèmes auxquels ils font face, causés par ce qu'ils estiment être une application exagérée de la loi

En ce qui a trait aux visiteurs de ces sites, rien n’indique qu'ils soient manipulés ou trompés de quelque façon que ce soit. Ils démontrent une certaine curiosité à l'égard de ces groupes et, dans le cas de ceux qui partagent leurs commentaires et leurs opinions, les signes de soutien sont évidents. À l'exception de quelques profils sur MySpace, les gangs de rue ne déploient aucun effort proactif pour inciter des individus à devenir membre de leur gang. Néanmoins, les sites de réseautage social offrent une nouvelle vitrine pour les personnes qui partagent ou sont sensibles aux valeurs sous-jacentes au mode de vie des gangs de rue.

Ce n'est pas la première fois que les médias et les efforts des organismes d'application de la loi sont ralentis par une perspective qui amplifie le côté stratégique des organisations criminelles et des membres de gangs (Morselli 2005). Dans plusieurs contextes, on tient pour acquis que les groupes criminels cherchent activement de nouveaux membres afin d'élargir leur horizon et gagner en puissance. Non seulement cette vision vient contredire les limites fondamentales en matière de confiance, de sécurité et de sélection des complices pour toute personne impliquée dans le milieu criminel, mais en plus, elle dissimule le scénario plus probable qu'il s'agit d'une geste essentiellement fortuit et non un plan stratégique . Les délinquants réputés sont plus susceptibles d'attirer des 
personnes que de chercher à attirer leur attention. Les aspirants criminels sont souvent les instigateurs de leur propre cooptation et pour chaque prétendant qui finit par joindre un gang, il y a une multitude d'admirateurs qui se contentent simplement d'observer les activités du gang de l'extérieur.

Même s'il est peu probable que les gangs se servent des sites de réseautage social pour recruter, l'émergence de ces sites remplit deux fonctions qui auront une incidence sur les groupes de criminels. Premièrement, il est allégué, depuis un certain temps, qu'un des principaux obstacles à l'expansion des groupes criminels est leur incapacité à diffuser leurs activités et leurs réalisations (Reuter 1983). Traditionnellement, les délinquants comptaient sur le bouche à oreille et sur l'attention que les médias leur consacraient pour établir et renforcer leur réputation. Ainsi, les résultats de la présente étude tendent à montrer que l'émergence des sites de réseautage social offre de nouveaux canaux permettant aux membres de groupes criminels de montrer leur image et de rehausser leur réputation. Deuxièmement, il est important de reconnaitre les interactions entre les membres des gangs de rue et les nombreux visiteurs de leurs sites de réseautage social comme étant une nouvelle forme de convergence (Felson 2003). Cependant, contrairement aux lieux physiques où les personnes se rassemblent pour interagir, les canaux fondés sur le Web, bien qu'ils prétendent à la présence d'«amis », font néanmoins converger des personnes qui demeurent distantes les unes des autres. Avant que ces canaux puissent servir au déploiement d'efforts de recrutement actif, ces interactions de base devront évoluer au-delà du simple échange d'images, de commentaires et d'opinions. Bien entendu, l'accès à un bassin aussi vaste d'observateurs curieux facilite tout processus de recrutement susceptible de dépasser la sphère d'Internet.

À bien des égards, les sites de réseautage social peuvent être d'excellentes sources de renseignements pour les organismes d'application de la loi. Ceux-ci, au cours des dernières années, ont commencé à exercer une certaine surveillance des gangs de rue sur les sites de réseautage social. Certains utilsent MySpace, Twitter et Facebook pour compiler des renseignements, cibler et solliciter de l'information sur des délinquants. Les services policiers ont aussi tiré avantage des réseaux sociaux afin de promouvoir leurs réalisations, encourager la dénonciation et diffuser leurs valeurs à un plus vaste public. Au Canada, des profils spécifiques ont été développés par les organismes d'application de la loi notamment à Montréal (Stevens 2009), Regina (Couture 2009) et Toronto (Masterman 2010). L'un des organismes les plus actifs dans l'utilisation de ces sites est Interpol qui s'en est récemment remis à Facebook pour retrouver 26 personnes impliquées dans les meurtres et l'exploitation sexuelle d'enfants.

Les enquêteurs des services de police se servent également des sites de réseautage social pour repérer des suspects. Ils utilisent des sites comme Facebook ou Twitter pour retracer l'adresse IP et les messages de différents suspects en espérant débusquer l'endroit où ils se cachent et traquer leurs 
activités (Muessig 2009). Ils compilent aussi des renseignements sur des cibles clés en effectuant le suivi du contenu placé en ligne par les membres des gangs de rue ou encore, en infiltrant les canaux virtuels. La surveillance de ces sites, sur une base régulière, permet de compiler des données sur les caractéristiques du réseau et des relations personnelles ou d'affaires des membres des gangs. Ces renseignements leur permettent de dresser une carte des réseaux sociaux de chaque individu et d'obtenir une évaluation plus détaillée des groupes et des organisations criminelles susceptibles de présenter un intérêt.

Aucune étude na encore réussi à mesurer l'efficacité et les effets que l'emploi des sites de réseautage social comme outil peut avoir sur le travail des organisations policières. Nous avons répertorié certains cas où la police a réussi à appréhender des délinquants, sans pour autant connaître le rôle exact qu'ont joué les sites de réseautage social dans ces arrestations. Nous en sommes donc aux premières étapes de cette expérimentation et, à l'instar de la population générale, les enquêteurs de police et les délinquants s'efforcent eux aussi de comprendre comment les sites de réseautage social peuvent contribuer à les aider à atteindre leurs objectifs respectifs. Bien que les réseaux sociaux continuent de capter l'attention des deux côtés de la loi, les organismes d'application de la loi doivent commencer à s'intéresser aux principaux domaines qui faciliteront leur progrès dans ce secteur.

Premièrement, il est recommandé que les organismes d'application de la loi et du renseignement établissent des liens plus formels avec les principaux administrateurs des sites de réseautage social. Ils doivent poursuivre leur apprentissage afin de comprendre de quelle façon ces canaux de communication sont structurés, comment les serveurs sont distribués, comment obtenir des renseignements sur les adresses IP et les conversations en privé et comment fermer une page ou y interdire l'accès. Des agents de liaison sont déjà en place entre les organismes d'application de la loi et d'autres sociétés de communications, en particulier en ce qui a trait aux services de téléphonie. Lorsqu'un mandat est lancé, les policiers savent habituellement de quelle façon procéder pour demander des renseignements relatifs aux conversations téléphoniques d'une personne. Les sites Web dominants comme Google peuvent aussi être sollicités pour aider les agents du renseignement criminel dans différentes opérations, mais aucun cadre opérationnel ne semble exister de façon systématique pour ces types de collaboration. À l'heure actuelle, il est nécessaire, d'élaborer un cadre clair et uniforme pour que ces échanges se poursuivent.

Deuxièmement, la surveillance des sites de réseautage social et sur Internet en général, semblent être le résultat de constatations fortuites ou de recherches massives effectuées par des personnes œuvrant au sein d'organismes d'application de la loi ou du renseignement criminel. Les percées technologiques ont suffisamment progressé pour permettre la mise en place d'outils de recherche automatisés plus systématiques et élaborés qui produisent des résultats et qui communiquent des alertes, au besoin. 
Troisièmement, il y aurait lieu à légiférer et à adopter des politiques en ce qui a trait aux enquêtes reliées à l'Internet. La venue de la toile mondiale a ouvert de vastes horizons où tous peuvent s'exprimer. Les services de police doivent donc composer avec des individus qui vivent dans des cadres législatifs et des territoires différents, mais qui convergent vers des communautés virtuelles communes. Tout comme d'autres formes de crime ou de déviances transnationales, des investissements plus importants devront être effectués dans le domaine de la collaboration policière et de l’adaptation des lois à cette nouvelle réalité sans frontières.

\section{Bibliographie}

BOYD, Danah et Nicole B. ELLISON. « Social Network Sites: Definition, History, and Scholarship », Journal of Computer-Mediated Communication, vol. 13 (2007).

BURT, Ronald S. «Structural Holes in Virtual Worlds », 2010. Document de travail disponible à 1' adresse suivante : http:/ /faculty .chicagobooth.edu/ronald. burt/research/SHVW. pdf

CRAIG, P. Software Piracy Exposed. Rockland (Massachusetts), Syngress Publishing, 2005.

COUTURE, Joe. « Policing Gang Activity in Regina», Leader-Post, 2010. Consulté à l'adresse suivante : http://www.leaderpost.com/news/Policing+gang+activity+Regina/2340360/story.html

DECKER, Scott H., Frank VAN GEMERT, et David C. PYROOZ. «Gangs, Migration, and Crime: The Changing Landscape in Europe and the USA », Revue de 1 'intégration et de la migration internationale = Journal of International Migration and Integration, vol. 10 (2009), p. 393-408.

FELSON, Marcus. Crime and Nature, Thousand Oaks, Sage, 2006.

FELSON, M.« The Process ofCo-offending », dans M.J. Smith et D.B. Cornish (Dir.) Crime Prevention Studies (p. 149-167), Monsey, Criminal Justice Press, 2003.

FLEISHER, Mark S. Beggars and Thieves: Lives of Urban Street Criminals, Madison, University of Wisconsin Press, 1995.

GAMBETTA, Diego. Codes of the Underworld: How Criminals Communicate, Princeton, Princeton University Press, 2009.

GUTIERREZ, Scott. «Street Gangs Using Internet for Violent Bragging Rites», Seattle PI, 2006. Consulté à l'adresse suivante: http:/ / www .seattlepi.com/local/277025 web gangs $10 . h t m l$

JACOBS, James. Gotham Unbound, New York, New York University Press, 1999. 
KATZ, Charles, Vincent J. WEBB et David R. SCHAEFER. «The Validity of Police Gang Intelligence Lists: Examining Differences in Delinquency Between Documented Gang Members and Nondocumented Delinquent Youth », PoliceQuarter/y, vol. 3 (2000), p. 413-437.

KENNEDY, David M., Anthony A. BRAGA et Anne M. PIEHL. «The (Un)Known Universe: Mapping Gangs and Gang Violence in Boston», dans David L. Weisburd et J. Thomas McEwen (Dir.) Crime Mapping and Crime Prevention (p. 219-262). New York, Criminal Justice Press, 1997.

KLEIN, Malcolm W. Street Gangs and Street Workers, Englewood Cliffs, Prentice-Hall, 1971.

KLEIN, Malcolm W. et Lois Y. CRAWFORD. «Groups, Gangs, and Cohesiveness », Journal of Research in Crime and Delinquency, vol. 4 (1967), p. 63-75.

MASTERMAN, Kevin. « Les médias sociaux mis à profit dans la répression du crime », Gazette- GRC, 2009. Consulté à l'adresse suivante: http://www.rcmpgrc.gc.ca/gazette/vol72n2/trend-tendancefra.htm

MCGLOIN, Jean Marie. «Police and Intervention Considerations of a Network Analysis of Street Gangs», Criminology and Public Policy, vol. 4 (2005), p. 607-635.

MORSELLI, Carlo. Inside Criminal Networks, New York, Springer, 2009.

MORSELLI, Carlo. Contacts, Opportunities, and Criminal Enterprise, Toronto, University of Toronto Press, 2005.

MORSELLI, Carlo, Mathilde TURCOTTE et Valentina TENTI. «La mobilité des groupes criminels», Rapport préparé pour Sécurité publique Canada, 2010.

MUESSIG, Ben. « Tweets is Watching-Street Gangs Using Twitter », Gothamist, 2009. Consulté à l'adresse suivante : http://gothamist.com/2009/11/29/street gangs use twitter to_plan th.php

NIELSEN COMPANY. « Led by Facebook, Twitter, Global Time Spent on Social Media Sites up 82\% Year over Year »,Nie/sen Wire, 2010. Consulté à l'adresse suivante:

http:/lblog.nielsen.com/nielsenwire/global/led-by-facebook-twitter-global-timespent-on-socialmedia-sites-up-82-year-over-year

REUTER, Peter. Disorganized Crime: The Economies of the Invisible Hand, Cambridge, MIT Press, 1983.

RIVLIN, Gary. « Wallflower At The Web Party», New York Times, 2006. Consulté à l'adresse suivante : http:/ /www.nytimes.com/2006/1 0/15/business/yourmoney/15friend.html? r=2 
SCRC. Rapport sur le crime organisé. Rapport préparé pour le Service canadien de renseignements criminels Canada, 2010.

SKOLNICK, Jerome H., Ricky BLUTHENTHAL et Theodore CORREL. « Gang Organization and Migration», dans S. Cummings et D.J. Monti (dir.) Gangs: The Origins and Impact ofContemporary Youth Gangs in the United States (p. 193-217). Albany, State University ofNew York Press, 1993.

STEVENS, Lauri.« Wanted in Montreal: One Social Media Cop », ConnectetdCOPS.net, 2010. Consulté à l'adresse suivante: http:/ /connectedcops.net/?p=453

TITA, George, K. Jack RILEY, Greg RIDGEWAY, Clifford GRAMMICH, Allan F. ABRAHAMSE et Peter W. GREENWOOD. Reducing Gun Violence: Results from an Intervention in East Los Angeles, Santa Monica, Rand, 2003.

VAN DE BUNT, Henk etC. VAN DER SCHOOT. Prevention ofOrganised Crime: A Situational Approach, Meppel, Dutch Ministry of Justice, 2003.

VAN DIJK, Jan. « Mafia Markers: Assessing Organized Crime and Its Impact Upon Societies »,Trends in Organized Crime, vol. 10 (2007), p. 39-56.

VASQUEZ, Joe. « Gangs Turn to Social Networking Sites to Recruit », CBS Crime Watch, 2008. Consulté à l'adresse suivante: http:/ /cbs5 .cornllocal/Intemet.gang.recruiting.2. 64803 8.html (Lien périmé) WEISEL, Deborah L. « The Evolution of Street Gangs: An Examination of Form and Variation », dans W.L. Reed et S.H. Decker (dir.) Responding to Gangs: Evaluation and Research (p.25-65). Washington DC, National Institute of Justice, 2002b.

WELLMAN, Barry. « The Global Village: Internet and Community », Ideas, vol. 1 (2004), p. 26-29.

WELLMAN, Barry. « Computer Networks as Social Networks », Science, vol. 293 (2001), p. 2031-2034.

WILLIAMS, Phil. « Organized Crime and Cybercrime: Synergies, Trends, and Responses »,Global Issues, vol. 6 (2001), p. 22-26.

WOMER, Sarah et Robert J. BUNKER. «Suriios Gangs and Mexican Cartel Use of Social Networking Sites», Small Wars and Insurgencies, vol. 21 (2010), p. 81-94.

ZHONGBAO, Kou et CHANGSHUI, Zhang. « Reply Networks On A Bulletin Board System», Physical Review, vol. 67 (2003).

\section{Tableau I}




\begin{tabular}{|l|l|}
\hline Groupes mentionnés & Groupes présents \\
\hline Mickey Cobras & Hells Angels \\
\hline & Shower Posse \\
& MS-13 \\
& Latin Kings \\
& Crips \\
\hline
\end{tabular}

\section{Tableau II}

Organisations criminelles

Hells Angels

MS-13

Crips

Bloods

Latin Kings

Almighty Vice Lord Nation

Shower Posse

14k Triad

Malvern Crew

18th Street

Mickey Cobras

Bad Boys

VVT

Wah Ching
Nombre d'admirateurs et de membres

14775

5923

4598

1993

1255

555

297

121

97

93

45

12

7

6 
Red Scorpions

Wo Shing Wo
6

5

\section{Tableau III}

\begin{tabular}{|c|c|c|}
\hline Organisations criminelles & Nombre d'amis & Nombre de page vues \\
\hline Hells Angels & 112748 & 83916 \\
\hline MS-13 & 5084 & \\
\hline Latin Kings & 4531 & 10090 \\
\hline Almighty Vice Lord Nation & 3541 & \\
\hline 18th Street & 1765 & 123605 \\
\hline Bloods & 1422 & \\
\hline Crips & 1279 & \\
\hline Asian Boys & 1261 & 6586 \\
\hline West Sidaz & 827 & \\
\hline Bad Boys & 715 & \\
\hline Wah Ching & 524 & 90985 \\
\hline West End Gang & 509 & \\
\hline Blood For Life & 354 & \\
\hline Red Scorpions & 54 & \\
\hline Malvern Crew & 39 & \\
\hline Galloway Boys & 2 & \\
\hline
\end{tabular}

